\title{
Canalis Sinuosos mimetizando reabsorção radicular: relato de caso
}

\section{Canalis sinuosus mimicking root resorption: case report}

\author{
Vanessa Sousa Nazaré Guimarães ${ }^{1}$ (D) Luciana Loyola Dantas ${ }^{2}$ (D) Juliana Gonzalez ${ }^{3}$ (D) , Iêda Margarida Crusoé Rocha Rebello ${ }^{4}$ (D) \\ Frederico Sampaio Neves ${ }^{4}$
}

1. Doutoranda em Patologia Humana pelo Instituto Gonçalo Moniz (FIOCRUZ), Salvador, BA, Brasil. 2. Doutoranda em Odontologia e Saúde da Universidade Federal da Bahia (UFBA), Salvador, BA, Brasil. 3. Radiologista de serviço privado em Salvador, BA, Brasil 4. Docente da Universidade Federal da Bahia (UFBA), Salvador, BA, Brasil

\section{Resumo}

Relato de caso: Relatar um caso de canal acessório do Canalis Sinuosos, localizado por palatino à raiz do dente 21 que, na radiografia panorâmica, foi observada como uma área radiolúcida sobreposta aos terços médio-apical dente 21, indicando reabsorção radicular, sendo indispensável uma investigação acurada por tomografia computadorizada de feixe cônico (TCFC). Conclusão: A TCFC se mostrou eficiente na identificação do Canalis Sinuosos em relação à radiografia panorâmica, possibilitando um correto diagnóstico para o caso.

Palavras-chave: Nervo maxilar. Variação Anatômica. Tomografia Computadorizada de Feixe Cônico.

\begin{abstract}
Case report: This report describes a case of Canalis sinuosus accessory canal, lingually to the root of the tooth 21 , that was observed on panoramic radiograph as a radiolucent area superimposed over the middle and apical thirds, indicating root resorption. However, cone-beam computed tomography (CBCT) recommended for an accurated diagnosis of the case disclose the presence of this anatomic variation. Conclusion: CBCT was more accurate in detection of Canalis sinuosus than panoramic radiography.
\end{abstract}

Key words: Maxillary Nerve, Anatomical variation, Cone beam computed tomography.

\section{INTRODUÇÃO}

O terço médio da face é inervado pelo nervo infraorbital, ramificação terminal do nervo maxilar, que é dividido em três ramos proximais (nervos alveolares superiores posterior, médio e anterior) $)^{1-3}$ e três ramos distais (palpebral inferior, nasal lateral e labial superior). 0 canal infraorbital emite um pequeno ramo, em sua face lateral, perto de seu ponto médio, para permitir a passagem do nervo alveolar superior anterior, denominado de Canalis Sinuosos ${ }^{1-4}$.

O Canalis Sinuosos inicia-se na cortical inferior da órbita, passando abaixo do forame infraorbitário para medial, em sentido à parede anterior do seio maxilar e contorna os limites laterais e inferiores da fossa nasal ${ }^{4}$. Ao final de seu trajeto, é comum haver bifurcações em canais acessórios com direção tanto para a região de assoalho de fossa nasal, como para a região de pré-maxila. Neste caso, o fim da ramificação maxilar pode variar na face palatina, vestibular ou transverso ao processo alveolar ${ }^{5}$. Desse modo, o nervo alveolar superior anterior, assim como as veias e as artérias correspondentes, emerge na região anterior da maxila para inervar os incisivos e caninos, bem como os tecidos moles adjacentes ${ }^{1,6}$.

As variações anatômicas da maxila raramente são descritas na literatura e, muitas vezes, a presença desses feixes vásculonervosos é desconhecida pelos profissionais ${ }^{2}$, o que pode resultar em complicações cirúrgicas que vão desde a perda da sensibilidade, até o maior risco de hemorragia ${ }^{7}$. No entanto, com o uso da Tomografia Computadorizada de Feixe Cônico (TCFC) na Odontologia e consequente incremento das informações obtidas $^{8}$, permitindo a identificação dessas variações anatômicas de forma mais fácil ${ }^{5,9}$. Portanto, este artigo tem por objetivo descrever a presença de uma ramificação acessória do Canalis Sinuosos que, nas imagens radiográficas, mimetizava um diagnóstico de reabsorção radicular.

\section{RELATO DE CASO}

O Paciente USN, sexo masculino, 44 anos de idade, compareceu à clínica privada de Radiologia Odontológica em Salvador, Bahia, Brasil para a realização de uma radiografia panorâmica (Carestream Health, Rochester, NY, EUA) para acompanhamento de tratamento ortodôntico. Foi observada a presença de uma área radiolúcida, pouco definida, sobreposta aos terços médio e apical da raiz do dente 21 , indicando um diagnóstico de reabsorção radicular (Fig. 1). 
Figura 1. Na radiografia panorâmica observa-se uma área radiolúcida sobreposta à raiz do dente 21 apontando para um diagnóstico de reabsorção radicular.



Para uma avaliação mais acurada, o paciente foi submetido ao exame por TCFC (Kodak 9000, Carestream Health, Rochester, NY, EUA) na região anterior da maxila. O corte axial (Fig. 2A),

coronal (Fig. 2C), sagital (Fig. 2D) e reconstrução tridimensional (Fig. 2B) evidenciaram uma ramificação acessória calibrosa do Canalis Sinuosos por palatino em relação à raiz do dente 21.

Figura 2. Imagens da TCFC evidenciando o corte axial (A), reconstrução tridimensional (B), corte coronal (C) e corte sagital (D) evidenciando a presença do canal acessório do Canalis Sinuosos localizado por palatino em relação ao dente 21.

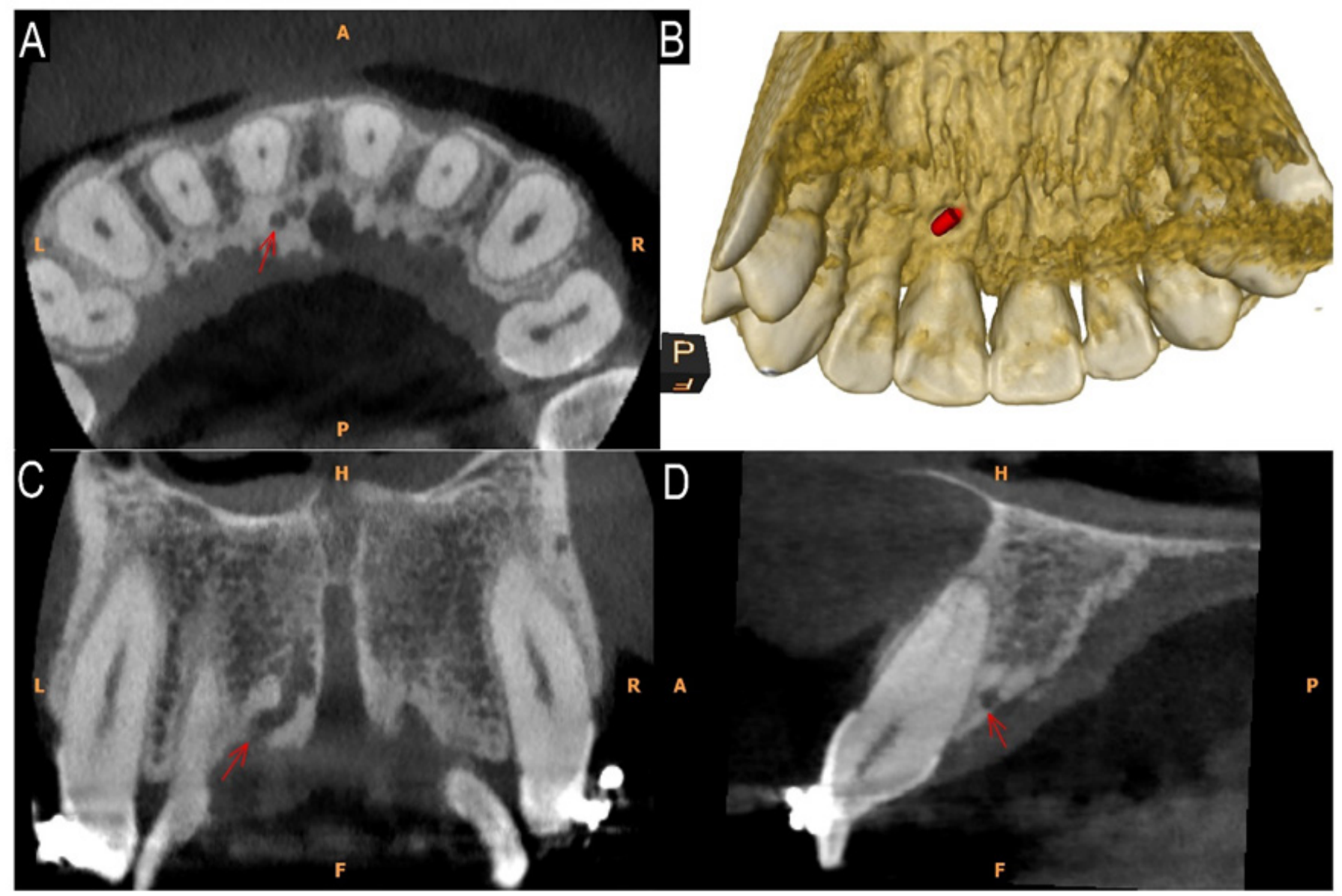




\section{DISCUSSÃO}

As estruturas anatômicas da porção anterior da maxila, como o Canalis Sinuosos, muitas vezes, são desconhecidas e negligenciadas em procedimentos clínicos. Somado a isso, a visualização dessa região é prejudicada em exames imaginológicos bidimensionais, devido à grande sobreposição de estruturas na formação da imagem, o que dificulta o diagnóstico nessa região. No entanto, com o advento da TCFC, permitiu que os profissionais pudessem avaliar essa região com maior acurácia, identificando variações anatômicas e possíveis patologias para uma conduta clínica adequada.

Os canais acessórios do Canalis Sinuosos raramente são observados em imagens bidimensionais, devido à porosidade de suas camadas corticais ou seu pequeno calibre que, geralmente, é inferior a $1 \mathrm{~mm}$ de diâmetro ${ }^{6,10}$. Em exceção, os Canalis Sinuosos mais calibrosos podem ser visualizados em radiografias, como uma área de menor densidade na região de incisivos e caninos superiores; porém, devido ao fato de o seu aspecto radiográfico ser pouco descrito na literatura, este pode mimetizar condições patológicas como lesões periapicais ${ }^{7}$ ou, assim como apresentado neste relato de caso, uma reabsorção radicular.

A TCFC permite uma avaliação tridimensional precisa da região anterior da maxila, fornecendo informações não apenas sobre quantidade e qualidade óssea, mas também sobre a identificação minuciosa de variações anatômicas ${ }^{10}$. Recentemente, Gurler et al. (2017) ${ }^{11}$ realizaram um estudo com 111 pacientes determinando a distância dos Canalis Sinuosos com caninos impactados nas imagens tomográficas e observaram a menor distância de 0,75 mm entre eles, enfatizando, assim, a necessidade desse exame para identificação dessa variação anatômica. A realização de uma TCFC foi indicada como a melhor conduta pré-operatória a fim de evitar danos ao nervo alveolar superior anterior e possíveis complicações como hiperestesia, parestesia e dores pós-cirúrgicas ${ }^{3}$.

Quanto à frequência da presença do Canalis Sinuosos, Machado et. al $(2016)^{6}$ realizaram uma pesquisa com 1.000 pacientes e, por meio de TCFC, observaram a presença do Canalis Sinuosos na maioria da população avaliada (521 pacientes - 52,10\%). No entanto, outros estudos com 500 e 100 pacientes apresentaram frequências de $36,20 \% 8$ e $88 \%{ }^{10}$. Em relação à localização, são observadas variações, podendo essa variação anatômica ser encontrada mais anteriormente, acima do incisivo central, ou, posteriormente, próximo ao canino de ambos os lados ${ }^{1,5,6,9,12}$. Além disso, o final da trajetória dos canais acessórios maxilares dos Canalis Sinuosos pode ocorrer por palatino, transverso ou vestibular aos dentes anterossuperiores, sendo a primeira mais comum, corroborando o caso descrito ${ }^{5}$.

No presente caso, na radiografia panorâmica, a imagem do Canalis Sinuosos sobrepôs-se à raiz do incisivo central superior esquerdo, mimetizando uma reabsorção radicular. Essa é uma consequência frequente ao tratamento ortodôntico, que acomete, principalmente, os dentes anteriores e, na maioria dos casos, é assintomática ${ }^{13}$. Radiograficamente, a reabsorção radicular se apresenta como um radiolucidez irregular na raiz do dente, presente em qualquer terço radicular. No entanto, a radiografia panorâmica é uma representação bidimensional de estruturas tridimensionais e apresentam limitações inerentes à sua técnica, como a sobreposição das estruturas anatômicas podendo simular essa condição patológica.

Diagnósticos de reabsorção radiculares equivocados provenientes da dificuldade na interpretação de radiografias convencionais não são incomuns na prática clínica. A natureza bidimensional dessas radiografias torna-a um método inadequado para determinar a localização, a gravidade e o tipo de reabsorção radicular ${ }^{14,15}$. Em adição, este estudo revela que variações anatômicas do Canalis Sinuosos também podem simular reabsorção radicular. Diante do exposto, este artigo reafirma a necessidade da realização de exames radiográficos tridimensionais para o correto diagnóstico em casos de suspeita de reabsorção radicular, como também para a identificação de variações anatômicas, especialmente na região anterior da maxila devido à presença de canais acessórios do Canalis Sinuosos.

\section{CONSIDERAÇÕES FINAIS}

Exames imaginológicos bidimensionais isolados não são indicados para a identificação de variações anatômicas na região anterior da maxila. Devido às limitações inerentes à técnica, essas informações podem ser subtraídas das imagens, como também simular condições adversas. Diante disso, é importante ressaltar a importância da TCFC para a identificação dessas variações e a sua relação com os tecidos adjacentes, permitindo um correto diagnóstico de patologias, planejamento cirúrgico e, consequentemente, contribuindo para o sucesso terapêutico.

\section{REFERÊNCIAS}

1. Neves FS, Crusoé-Souza M, Franco LC, Caria PH, Bonfim-Almeida P,CrusoéRebello I. Canalis sinuosus: a rare anatomical variation. Surg Radiol Anat. 2012 Aug; 34(6): 563-6. doi: 10.1007/s00276-011-0907-6.

2. Oliveira-Santos C, Rubira-Bullen IR, Monteiro SA, León JE, Jacobs R. Neurovascular anatomical variations in the anterior palate observed on CBCT images. Clin Oral Implants Res. 2013 Sep;24(9):1044-8. doi: 10.1111/j.16000501.2012.02497.x.

3. Olenczak JB, Hui-Chou HG, Aguila DJ 3rd, Shaeffer CA, Dellon AL, Manson
PN. Posttraumatic Midface Pain: Clinical Significance of the Anterior Superior Alveolar Nerve and Canalis Sinuosus. Ann Plast Surg. 2015 Nov;75(5): 543-7. doi: $10.1097 /$ SAP.0000000000000335.

4.von Arx T, Lozanoff S. Anterior superior alveolar nerve (ASAN). Swiss Dent J. 2015; 125(11): 1202-9.

5. Machado VC, Chrcanovic BR, Felippe MB, Manhães LR Júnior, Carvalho PS. Assessment of accessory canals of the canalis sinuosus: a study of 1000 cone beam computed tomography examinations. Int J Oral Maxillofac Surg. 2016 
Dec; 45(12): 1586-1591. doi: 10.1016/j.ijom.2016.09.007.

6.Torres MG, Faro Valverde L, Vidal MT, Crusoé-Rebello IM. Branch of the canalis sinuosus: a rare anatomical variation--a case report. Surg Radiol Anat. 2015 Sep;37(7):879-81. doi: 10.1007/s00276-015-1432-9.

7. Shelley AM, Rushton VE, Horner K. Canalis Sinuosos mimicking a periapical inflammatory lesion. Br Dent J. 1999 Apr; 186(8): 378-9. PubMed PMID: 10365458.

8. Costa RBA, Coelho IWF, Carvalho FB, Crusoé-Rebello I, Neves FSN. Avaliação clínica, radiográfica e tomográfica de fraturas radiculares: série de casos. J Health Biol Sci. 2019 Abr-Jun; 7(2): 172-176.

9. Manhães LR Júnior, Villaça-Carvalho MF, Moraes ME, Lopes SL, Silva MB, Junqueira JL. Location and classification of Canalis sinuosus for cone beam computed tomography: avoiding misdiagnosis. Braz Oral Res. 2016; 30(1): e49. doi: 10.1590/1807-3107BOR-2016.vol30.0049.

10. von Arx T, Lozanoff S, Sendi P, Bornstein MM. Assessment of bone channels other than the nasopalatine canal in the anterior maxilla using limited cone beam computed tomography. Surg Radiol Anat. 2013 Nov; 35(9): 783-90. doi: 10.1007/s00276-013-1110-8.

11. Gurler G, Delilbasi C, Ogut EE, Aydin K, Sakul U. Evaluation of the morphology of the canalis sinuosus using cone-beam computed tomography in patients with maxillary impacted canines. Imaging Sci Dent. 2017 Jun; 47(2): 69-74. doi: 10.5624/isd.2017.47.2.69.

12. Wanzeler AM, Marinho CG, Alves Junior SM, Manzi FR, Tuji FM. Anatomical study of the canalis sinuosus in 100 cone beam computed tomography examinations. Oral Maxillofac Surg. 2015 Mar; 19(1): 49-53. doi: 10.1007/ s10006-014-0450-9.

13. Agarwal SS, Chopra SS, Kumar P, Jayan B, Nehra K, Sharma M. A radiographic study of external apical root resorption in patients treated with single-phase fixed orthodontic therapy. Med J Armed Forces India. 2016 Dec; 72(Suppl 1): S8-S16. doi: 10.1016/j.mjafi.2016.04.005.

14. Takeshita WM, Chicarelli M, Iwaki LC. Comparison of diagnostic accuracy of root perforation, external resorption and fractures using cone-beam computed tomography, panoramic radiography and conventional \& digital periapical radiography. Indian J Dent Res. 2015 Nov-Dec; 26(6): 619-26. doi: 10.4103/0970-9290.176927.

15. Lima TF, Gamba TO, Zaia AA, Soares AJ. Evaluation of cone beam computed tomography and periapical radiography in the diagnosis of root resorption. Aust Dent J. 2016 Dec; 61(4):425-431. doi: 10.1111/adj.12407.

\section{Como citar este artigo/How to cite this article:}

Guimarães VSN, Dantas LL, Gonzales J, Rebello IMCR, Neves FS. Canalis sinuosos mimetizando reabsorção radicular: relato de caso. J Health Biol Sci. 2019 Jul-Set; $7(3): 320-323$ 